\title{
Conditioning copulatory behavior to an artificial object: Efficacy of stimulus fading
}

\author{
MICHAEL DOMJAN, MARILYN HUBER-MCDONALD, and KEVIN S. HOLLOWAY \\ University of Texas, Austin, Texas
}

\begin{abstract}
Copulatory behavior rarely occurs in response to an arbitrary inanimate object. However, such behavior can provide important information about the stimulus control of copulatory behavior. In the present study, male Japanese quail were administered $15-20$ conditioning trials that in cluded exposure to an inanimate object and an opportunity to copulate with a live female quail. For some subjects, the stimulus object was always entirely covered with terrycloth. For other birds, the stimulus object contained a taxidermically prepared head and neck of a quail hen. Fading consisted of gradually covering up the neck and then the head portions of the stimulus object over successive trials. After conditioning, all subjects were tested with the entirely covered object. The fading procedure facilitated the conditioning of copulatory behavior to the entirely artificial object. In subjects that received the fading procedure, learning did not depend on pairings of the stimulus object with copulatory opportunity. The results are discussed with respect to an associative mediation mechanism.
\end{abstract}

Species-typical responses occur in a predictable order. A central issue in the analysis of species-typical behavior is whether responses that occur early in a behavioral sequence are fundamentally different from responses that occur later in the sequence. In classical ethological theory, actions that occur early in a sequence are referred to as "appetitive" responses, and actions that occur at the end of the sequence are referred to as "consummatory" responses. Appetitive responses are assumed to be involved in the search for releasing stimuli and have been characterized as variable, purposive, and "adaptable to changing situations" (Eibl-Eibesfeldt, 1970, p. 45). In contrast, consummatory responses are involved in the completion of the behavioral sequence and have been characterized as more stereotyped and "automatically discharged" (Eibl-Eibesfeldt, 1970, p. 45).

Characterizations of appetitive and consummatory responses as "adaptable to changing situations" and "automatically discharged," respectively, imply differences in the susceptibility of these responses to learning. Appetitive responses are presumed to be more easily influenced by past experience than are consummatory responses. Tinbergen $(1951$, p. 105), for example, noted that appetitive responses exhibit "plasticity," whereas consummatory responses are "rigid."

These presumed differences in the susceptibility of appetitive and consummatory behavior to learning or experiential modification may be investigated in sexual behavior systems, in which appetitive behavior consists of

This research was supported by Grant MH 39940 from the National Institute of Mental Health. We thank Fawn McDonald, and Chris Fox for help in running the subjects and Mitch Leben for help in scoring data. Requests for reprints should be addressed to $M$. Domjan, Department of Psychology, University of Texas, Austin, TX 78712. searching for and approaching a potential sexual partner and consummatory responses consist of mounting and copulation. Successful systematic demonstrations of the conditioning of appetitive sexual behavior are available in both fish and avian species. Hollis, Cadieux, and Colbert (1989), for example, observed that in blue gouramis, the pairing of a conditioned stimulus (a red light) with visual access to a conspecific of the opposite sex results in conditioned approach and display responses to the red light. Sexually conditioned approach responses to arbitrary visual conditioned stimuli also have been observed in male Japanese quail (Domjan, Lyons, North, \& Bruell, 1986).

Consistent with the suggestion that appetitive behavior is more amenable to conditioning than is consummatory behavior, conditioned copulatory responses were not observed in the studies with gouramis and quail noted above. This may have been because of the type of conditioned stimuli (localized visual cues) that were used. Such conditioned stimuli may not have provided the supportive stimulation that may be necessary for mounting and copulatory contact responses.

Conditioning of consummatory sexual behavior may be more likely if the conditioned stimulus is a three-dimensional (3-D) object that the subjects can mount and copulate with more easily. Some isolated observations of copulatory behavior directed toward arbitrary 3-D objects have been reported. For example, Young (1949) described a male eastern robin that copulated with a clump of earth and a piece of crumpled newspaper. Barraud (1953) reported observing a young male house sparrow copulating with a dandelion. Ficken and Dilger (1960) described a male Fischer's lovebird copulating with a knot on a wood perch, a woodthrush copulating with a smooth stone, and an American redstart copulating with a clump of dried droppings. Beach (1950) reported that dogs masturbated by 
an experimenter will come to direct their sexual behavior toward that experimenter, and Michael (1961) described an instance of a male cat copulating with a toy. These anecdotal observations are provocative; however, they involved very few subjects (one per species in most cases) and provided only hints about what factors might have been responsible for the observed behavior.

Domjan, O'Vary, and Greene (1988, Experiments 3 and 4) attempted to condition copulatory behavior to an arbitrary stimulus object in male Japanese quail by using procedures that had been found to be successful in other forms of sexual conditioning with this species. The conditioned stimulus was a small, bright yellow, stuffed toy dog (a Pound Puppy), propped up so that its head was about the height of a squatting female quail's head. The subjects received conditioning trials consisting of exposure to the stuffed toy for $30 \mathrm{sec}$ immediately before access to a female quail, with which the subjects invariably copulated. For a control group, copulation with a quail hen was provided about $90 \mathrm{~min}$ before exposure to the stuffed toy. Consistent with other demonstrations of the conditioning of appetitive sexual behavior, pairings of the stuffed toy with copulatory opportunity resulted in a rapid acquisition of conditioned approach to the toy. However, the stuffed toy never came to elicit copulatory behavior.

In contrast to the lack of systematic evidence of the conditioning of copulatory behavior to arbitrary 3-D objects, copulatory behavior has been observed in response to 3-D objects that have sign stimulus features. For example, male copulatory behavior has been observed in response to taxidermic models of female conspecifics in a variety of bird species such as chicken, turkey, cowbird, ruffed grouse, and blackbird (Allen, 1934; Carbaugh, Schein, \& Hale, 1962; Noble \& Vogt, 1935; Schein \& Hale, 1965; Schoettle \& Schein, 1959). These observations were derived from the behavior of sexually experienced male birds. This leaves open the possibility that some of the copulatory responding was a product of prior sexual learning. Consistent with this possibility, studies with male Japanese quail have demonstrated that prior sexual experience increases the likelihood that males will copulate with a model that contains the head and neck of a female quail (Domjan, Greene, \& North, 1989). Male Japanese quail also can be conditioned to copulate with a quail hen adorned with bright orange feathers by pairing exposure to an adorned hen with the opportunity to copulate with a normal female quail (Domjan et al., 1988). However, the conditioned responding is lost if the orange feathers are presented alone, in the absence of sign stimulus features of a female quail.

The above evidence suggests that there are serious constraints on the conditioning of consummatory sexual behavior. Studies with taxidermic models have shown that the presence of a live sexually receptive female is not necessary for the occurrence of male copulatory behavior. However, copulatory behavior in the absence of some species-typical female cues has been observed only in isolated anecdotal reports.
The purpose of the present study was to explore further the possibility of conditioning copulatory responses to an arbitrary stimulus object. Japanese quail were used in the experiments to take advantage of prior knowledge about the conditions that promote copulatory behavior in this species. Because previous research had indicated that male quail will copulate with models that contain female features, our strategy was to establish responding to a stimulus object that had quail features in the initial stages of training. The female features then were removed gradually, using a variation of fading procedures that have been successfully employed in the conditioning of stimulus discriminations (e.g., Terrace, 1966). The quail features that we employed, on the basis of our previous findings of the importance of cues of a hen's head and neck in stimulating male sexual behavior (Domjan et al., 1989; Domjan \& Nash, 1988; Domjan et al., 1988) were those of the head and neck of a female bird.

\section{EXPERIMENT 1}

Two groups of subjects were tested in Experiment 1. On each trial, an inanimate object with which the subjects could copulate was presented for $30 \mathrm{sec}$, followed immediately with access to a live sexually receptive quail hen. For birds in the fading group, the inanimate object during the first few conditioning trials consisted of a taxidermic specimen containing the head and neck of a female quail mounted vertically in front of a soft mounting pad covered with beige terrycloth (see Object 1 in Figure 1). Once copulation occurred with the head-and-neck model, the neck and then the head were covered up gradually over successive trials with beige terrycloth. Eventually, no quail features remained, and the inanimate object consisted of a vertical terrycloth protrusion with a terrycloth mounting pad behind it (Object 6 in Figure 1). Group 2 received a nonfading procedure in which the entirely covered object (Object 6 in Figure 1) was presented on each trial. At the end of the experiment, both groups were tested with the entirely artificial object.

\section{Method}

Subjects. Forty adult male experimentally naive Japanese quail (Coturnix coturnix japonica), 2.5-5 months old, served as subjects. The birds were hatched from eggs obtained from a colony maintained at the University of Texas at Austin. At 4-5 weeks of age, they were moved from mixed-sex brooders and housed in metal quail cages (G.Q.F. Manufacturing Co., Savannah, GA). Male birds were individually housed, and female birds were housed in groups of up to 4 per cage. Lights in the laboratory were on from 6 a.m. to 10 p.m. daily, which provided ample photostimulation to maintain the birds in reproductive readiness. Food (turkey grower) and water were available continuously.

Prior to participating in the experiment, all of the subjects were pretested for copulatory behavior by having a female quail introduced into their home cage for $5 \mathrm{~min}$. This is usually enough time for Japanese quail to complete the copulatory response sequence (Schein, Diamond, \& Carter, 1972). Only subjects that made cloacal contact with the female bird during the pretest were selected to participate in the experiment. As it turned out, not all of the sub- 

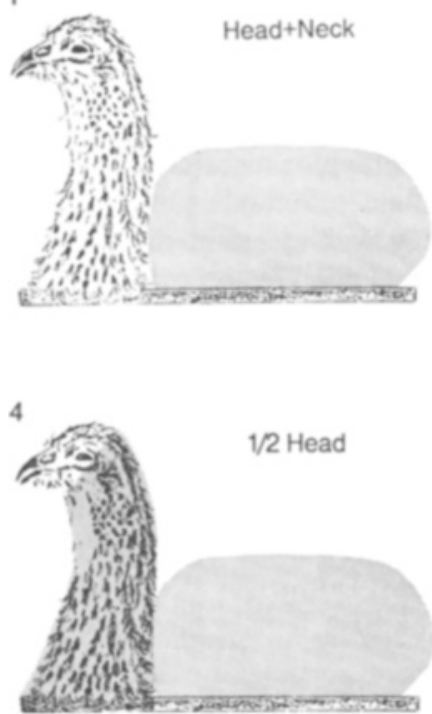

2
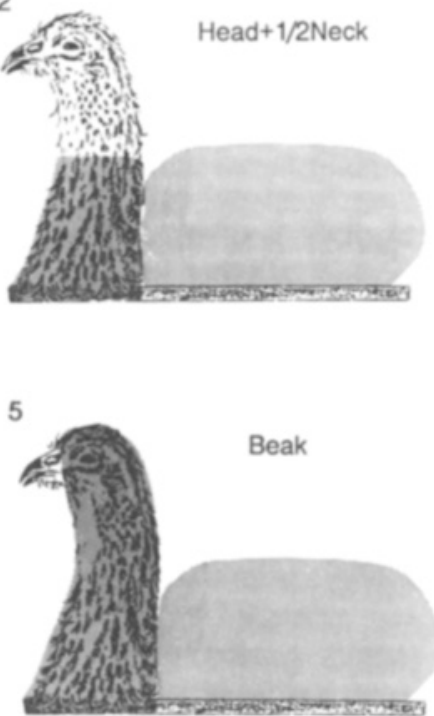

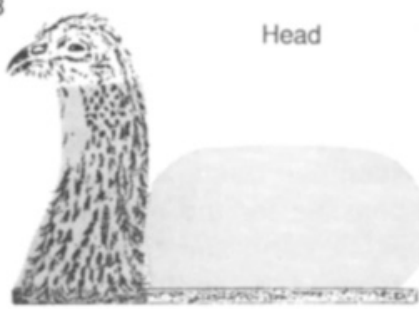

$3 \mathrm{~cm}$

6

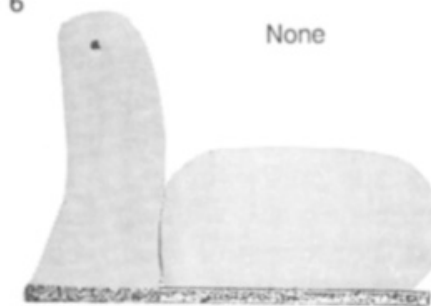

Figure 1. The stimulus objects that were used. Objects 1-5 consisted of the head and neck of a quail hen that had been taxidermically prepared and mounted vertically in front of a terrycloth pad filled with polyester fiber. The neck and head portions of Objects 2-5 were partially covered with terrycloth, as indicated by the stippled shading. For Object 6, the quail head and neck were replaced with a vertical terrycloth cylinder, which was filled with polyester fiber.

jects that copulated in the home cages copulated in the test arenas (see below).

An equal number of female quail of similar age were used during periods of copulatory opportunity. Each male bird was paired with a female and encountered the same quail hen during each period of copulatory opportunity.

Apparatus. The experimental procedures were conducted in large observation chambers $(91.4 \mathrm{~cm}$ wide $\times 106.7 \mathrm{~cm}$ high $\times 121.9 \mathrm{~cm}$ deep; see Figure 2). The two sides and the top of the chambers were made of sealed plywood; the front and back walls and the floor were made of wire mesh. The middle of one side wall had a small opening $(14.0 \times 15.5 \mathrm{~cm})$, which provided access to a small side cage $(14.0 \times 15.5 \times 11.5 \mathrm{~cm})$. The side cage served as a holding area for the birds before a conditioning trial and was usually blocked off by a vertically sliding masonite panel that served as a door.

On the wall directly opposite the side cage, a release box $(12.8 \times$ $20.3 \times 12.8 \mathrm{~cm}$ ) rested on brackets $21 \mathrm{~cm}$ above the floor. This box was used to provide access to a female quail paired with exposure to a stimulus object. The sides of the release box were made of sealed wood, and the top was wire mesh. The top was attached to a string passed over a pulley to allow the experimenter to raise and lower the box. The floor of the release box was hinged, so that when the box was raised, the floor swung open, and the quail hen dropped into the test arena. During trials, the inanimate stimulus object with which subjects could copulate was placed directly below the release box facing the middle of the chamber at $45^{\circ}$ relative to the side wall. The floor of the experimental chamber was marked in nine areas of equal size. One of these was centered around the stimulus object.

A series of inanimate stimulus objects was used (see Figure 1). Objects 1-5 consisted of the head and neck of a quail hen that had been taxidermically prepared and mounted vertically in front of a terrycloth pad filled with polyester fiber. For Object 6, the quail head-and-neck section was replaced with a closed vertical terrycloth cylinder, which was also filled with fiber. A black eyespot was painted on both sides of this vertical section, but informal tests with and without the eyespots at the end of the experiment suggested that they were inconsequential.
Procedure. The experiment was conducted in two equal counterbalanced replications. In each replication, the subjects were assigned to one of two squads of 10 subjects each. Each squad was housed in the experimental chambers during alternate 24 -h periods. The subjects were exchanged around noon each day, with the birds that had been in the experimental chambers moved to the wire-mesh home cages and those in the home cages moved to the experimental chambers. This trade-out procedure was started 18-25 days before any experimental procedures and continued throughout the experiment. Trials were conducted in the mid-morning and mid-afternoon. The use of the alternating housing procedure resulted in successive trials for a given bird being alternated between morning and afternoon trials.

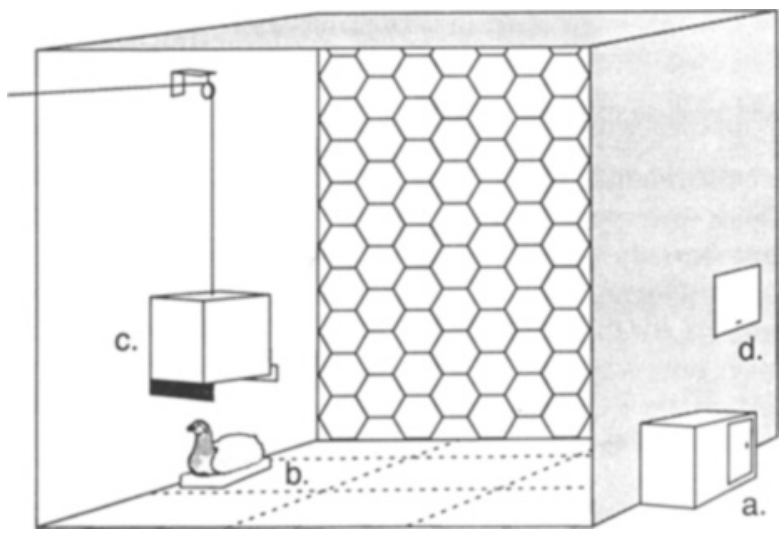

Figure 2. A diagram of the experimental chamber: $a=$ holding compartment in which the male subject was placed before each conditioning trial, $b=$ stimulus object, $c=$ release box from which a female quail was released into the experimental chamber on paired trials in Experiments 1-3, and $d=$ side door for placing a female quail into the experimental chamber, used only in the unpaired procedure in Experiment 2. 
Before the start of conditioning trials, the subjects were habituated to being placed in the side cages. On seven to eight occasions, each subject was moved from the experimental chamber to the side cage, with the door to the side cage closed. The door was then opened, releasing the subject back into the experimental chamber In addition, on three occasions, the subjects in the second replication received access to a live female quail for $5 \mathrm{~min}$ in the experimental chambers. This was expected to facilitate their responding to the models (Domjan et al., 1989).

Conditioning trials were conducted after the habituation phase At the start of each trial, the male subject was placed into the side compartment, with the side door closed. A quail hen was then placed into the release box, and a stimulus object was placed beneath the box. After 5-10 $\mathrm{min}$, the male was released into the experimental chamber; $30 \mathrm{sec}$ later, the quail hen was released, and the 2 birds were allowed to interact for $5 \mathrm{~min}$, during which copulation usually occurred. The quail hen and the stimulus object were then removed to bring the trial to an end. Thus, the stimulus object was paired with copulatory opportunity.

For the subjects in Group NP1 (Nonfading, Paired, Experiment 1. $n=20$ ), Object 6 (see Figure 1), which was made entirely of terrycloth filled with polyester fiber, was presented on each conditioning trial. For the subjects in Group FPI (Fading, Paired, Experiment $1, n=20$ ), a series of different stimulus objects was presented. For the FP1 subjects in the first replication, the headand-neck object (Object 1 in Figure 1) served on each of the first 10 trials. After that, the quail head-and-neck cues were gradually covered up according to the performance of each subject. If the subject made a cloacal contact response with an object on 2 consecutive trials (e.g., Object 2), the next object in the sequence was presented on the next trial (Object 3). Occasionally, if a subject was responding vigorously, the stimulus sequence was advanced after only 1 trial of copulation with an object. If copulation failed to occur, the sequence was backed up to the last object with which the subjects copulated.

A total of 20 conditioning trials were conducted for each group in the first replication. In the second replication, the fading sequence for Group FPl was started after the 5th conditioning trial, and a total of 15 conditioning trials were conducted for both the fading and the nonfading subjects. Six of the subjects in Group FP1 in the first replication and 5 of them in the second replication completed the fading sequence by the end of the conditioning trials. By the end of the conditioning trials, the remaining subjects in Group FPI were making little progress in the stimulus sequence. Because the two replications provided similar results, the acquisition data from the first $\mathbf{1 5}$ trials of each replication were pooled.

After the conditioning phase, each subject was tested with $\mathrm{Ob}$ ject 6 , which was made entirely of terrycloth filled with polyester fiber. This test trial was conducted in the same manner as were the conditioning triais except that a female hen was not placed in the release box above the stimulus object, and the subjects were permitted to interact with the object for $5 \mathrm{~min}$.

Because we were interested in male copulatory behavior, we only used data from the subjects that copulated with the live quail hens in the experimental chambers during the conditioning trials. Although the subjects were pretested for copulatory behavior in the home cages, 2 birds in Group FP1 and 3 in Group NP1 never copulated during the conditioning trials. In addition, 1 subject in Group FP1 broke its leg before the end of the experiment. These losses left 17 subjects in each group.

Response measures. The interactions of the subjects with the stimulus objects were recorded on videotape for the first $30 \mathrm{sec}$ of each conditioning trial (before the live female quail was introduced) and for the 5-min test trial with the completely terrycloth stimulus at the end of the experiment. The video records were then scored with respect to five response categories. The first of these was time spent in that ninth of the test arena in which the stimulus object was located (see Figure 2). This provided a measure of ap- proach and proximity to the stimulus object (and the location where the female was released for the paired groups). The second response was pecks, and the remaining three responses involved components of copulatory behavior. Copulatory behavior in male quail consists of the male grabbing the back of the hen's head or neck, mounting the hen's back with both feet, and then making cloacal contact movements (Wilson \& Bermant, 1972). A grab was scored if the subject grabbed the feathers or terrycloth surface of the stimulus object. Grabs were distinguished from pecks by whether the subject held onto the surface of the stimulus object. A peck was scored if the subject hit the object with its beak and quickly withdrew without holding onto the terrycloth. A mount was scored if the subject got on top of the stimulus object with both feet. A cloacal contact response was scored if the subject arched its back and appeared to bring its cloaca in contact with the back of the stimulus object. The cloacal contact response was often accompanied by hythmic shaking of the cloacal area when the bird was in the arched position.

The conditioning and test trials were scored first by one of the authors (M.M. or M.D.). Subsequently, all of the test sessions were scored by an additional observer (M.L.), who was unaware of the group assignment of the subjects. Correlations between the results of the two observers who scored the test data were computed for each replication of Experiment 1. For the first replication, these correlations were $.99, .99, .90, .97$, and .82 for pecks, time near the stimulus objects, grabs, mounts, and cloacal contacts, respectively. For the second replication, these correlations were .99 for each response. Because of the high value of these reliability correlations, only results from the original scoring of the videotapes are presented.

\section{Results}

Acquisition: Noncopulatory response measures. The frequency with which the subjects pecked the stimulus objects during the first 15 conditioning trials is presented in the top panel of Figure 3. The subjects that received the fading sequence of stimulus objects (which included quail features) showed more pecking behavior than did Group NP1 $[F(1,32)=10.65, p<.01]$. However, neither the main effect of trials nor the trials $\times$ group interaction was significant $(F s<1.3)$. These results suggest that the conditioning procedures did not influence pecking behavior. Stimulus objects that contained quail features were simply more effective in eliciting pecking than was the entirely terrycloth object that was presented to the subjects in group NP1.

The amount of time the subjects spent near the stimulus objects is presented in the lower panel of Figure 3. Both groups showed an increase in time spent near the stimulus objects as conditioning proceeded $[F(14,448)=$ $12.66, p<.01]$. The fading group (FP1) spent more time near the stimulus objects than did the nonfading group (NP1) $[F(1,32)=16.31, p<.01]$. However, the groups $X$ trials interaction was not significant $(F<1.0)$, indicating that the course of acquisition of approaching and remaining near the stimulus objects was not significantly different in the two groups.

Acquisition: Copulatory response measures. The connected points in Figure 4 show the frequency of grab, mount, and cloacal contact responses directed toward the stimulus objects that occurred during the course of acquisition. Similar results were obtained with each of these response measures. The subjects that received the fading 

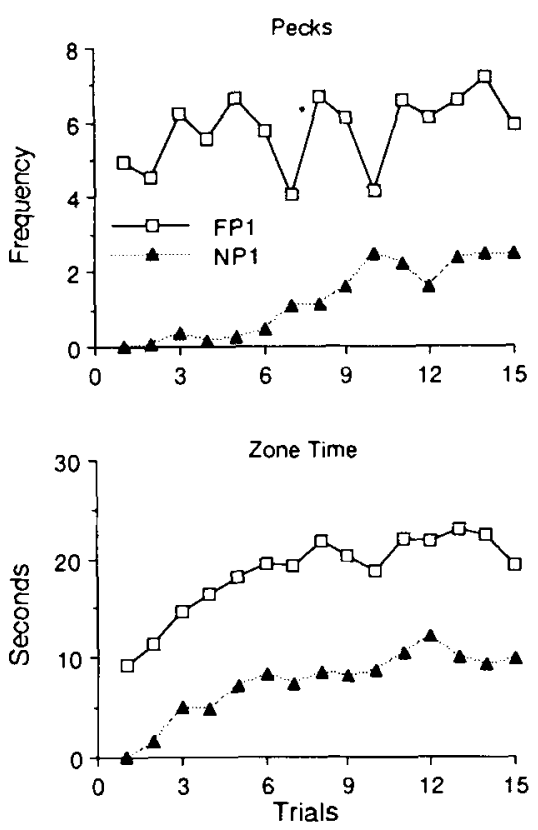

Figure 3. The mean frequency of pecks (top panel) and mean seconds near the stimulus objects during the first 15 acquisition trials of Experiment 1. (Group FP1 received a fading paired procedure; Group NP1 received a nonfading paired procedure.)

sequence of stimulus objects (Group FP1) increased their responding as training progressed. In contrast, little change was evident in the responses of the subjects that received the entirely artificial stimulus object on each trial (Group NP1). In addition, the subjects in the nonfading group were much less likely to make grab, mount, and cloacal contact responses with their stimulus object than were subjects in the fading group.

A groups $\times$ trials analysis of variance (ANOVA) was calculated for each response measure. Each of these analyses indicated a significant effect of groups $[F \mathrm{~s}(1,32)=$ $16.28,17.68$, and 15.07 , for the grab, mount, and cloacal contact responses, respectively, all $p s<.01]$. The analyses also indicated significant effects of trials for each response measure $[F \mathrm{~s}(14,448)=4.47,5.19$, and 4.67 , for the grab, mount, and cloacal contact responses, respectively, all $p s<.01]$. In addition, the groups $\times$ trials interaction was significant for each response measure $\left[F_{\mathrm{s}}(14,448)=1.95,2.78\right.$, and 2.62 , all $\left.p \mathrm{~s}<.05\right]$. These latter findings indicate that the subjects exposed to the fading sequence of models increased their copulatory responses to the stimulus objects to a significantly greater extent across trials than did the subjects in Group NP1.

Postconditioning test: Copulatory response measures. During the test trial, both the fading and the nonfading groups were exposed to the entirely artificial stimulus object (Object 6, Figure 1). This permitted comparing their performance under identical conditions and in response to an object lacking the species-typical plumage of a quail hen. The mean frequency of grab, mount, and cloacal contact responses during the final test session are shown by the unconnected points to the right of each acquisition curve in Figure 4. The greater levels of responding that were evident in Group FPl, as compared with Group NP1, during the training trials were also evident in the final test session. The subjects that received the fading sequence of stimulus objects made more grab, mount, and cloacal contact responses during the final test session than did the subjects in the nonfading group.

The carryover of responding from the end of acquisition to the test session was evaluated for each response measure by separate ANOVAs that included Trial 15 of acquisition and the test session for each group. These analyses indicated that the subjects that received the fading procedure responded more than those that received the nonfading procedure $\left[F_{\mathrm{s}}(1,32)=4.34,5.32\right.$, and 5.23 , for the grab, mount, and cloacal contact responses, respectively, all $p s<.05]$. The interaction between groups and the shift from acquisition to testing was not significant for any response measure (all $F \mathrm{~s}<1.0$ ). This latter outcome confirms that the group differences that were evident at the end of acquisition were not altered significantly
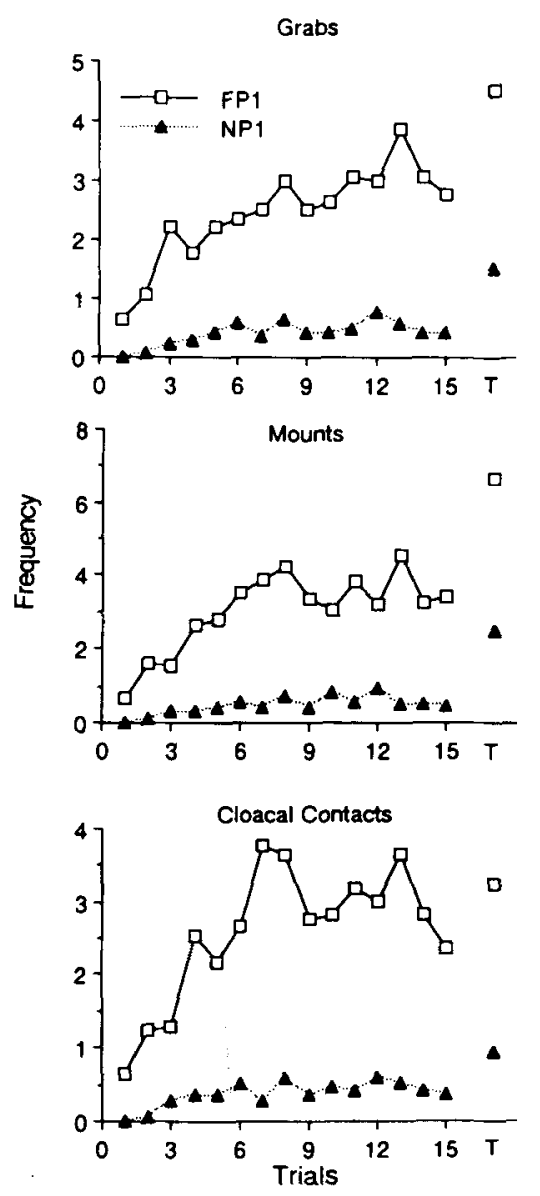

Figure 4. Mean frequencies of grab, mount, and cloacal contact responses elicited by the stimulus objects during the first 15 conditioning trials (connected points) and the postconditioning test (the unconnected points on the right of each panel) in Experiment 1. (Group FP1 received a fading paired procedure; Group NP1 received a nonfading paired procedure.) 
Table 1

Proportion of Subjects Making at Least One Grab, Mount, or Cloacal Contact Response to the Fully Artificial Model During the Postconditioning Test

\begin{tabular}{lccc}
\hline & \multicolumn{3}{c}{ Response } \\
\cline { 2 - 4 } & Grab & Mount & Cloacal Contact \\
\hline Group FP1 & \multicolumn{3}{c}{ Experiment 1} \\
Group NP1 & $9 / 17$ & $10 / 17$ & $8 / 17$ \\
& $3 / 17$ & $4 / 17$ & $3 / 17$ \\
Group FP2 & \multicolumn{3}{c}{ Experiment 2} \\
Group FU2 & $5 / 10$ & $4 / 10$ & $4 / 10$ \\
& $6 / 10$ & $6 / 10$ & $4 / 10$ \\
Group NP3 & \multicolumn{3}{c}{ Experiment 3} \\
& $3 / 13$ & $2 / 13$ & $1 / 13$ \\
Fading & \multicolumn{4}{c}{ Overall } \\
Nonfading & $20 / 37$ & $20 / 37$ & $16 / 37$ \\
\hline
\end{tabular}

by the introduction of the fully artificial stimulus object for all subjects during the test session.

The number of subjects that made at least one grab, mount, or cloacal contact response to the entirely artificial model during the final test is summarized in Table 1 . The subjects that received the fading sequence of models during training were 3 times more likely to make at least one grab response to the artificial stimulus object than were the subjects that received the nonfading sequence of models. Nine of the 17 subjects in Group FP1 grabbed the model, whereas only 3 of the 17 subjects in Group NP1 did so. Similar, but somewhat smaller, differences between the groups were evident in the frequency of subjects making mount and cloacal contact responses to the stimulus object.

\section{Discussion}

The present findings provide systematic evidence of the development of copulatory responding to an inanimate object that has minimal species-typical features. In contrast to previous anecdotal accounts of a few unusual subjects that were observed to copulate with artificial stimuli, about $50 \%$ of the subjects that received the fading sequence of models in the present experiments ended up making grab, mount, and cloacal contact responses to the artificial stimulus object. However, the present findings do not encourage rejection of the claim that consummatory behavior is less susceptible to modification by learning than is appetitive behavior (e.g., Eibl-Eibesfeldt, 1970; Tinbergen, 1951). Consistent with that claim, the subjects that were exposed to the entirely artificial stimulus object on every conditioning trial (Group NP1) approached and spent increasing amounts of time near the object as training progressed (see Figure 3), but they did not show substantial increases in grab and cloacal contact responses (Figure 4).

The present findings provide some suggestions about factors that might have promoted copulation with a stimulus object having minimal species-typical cues. As in previous research (e.g., Domjan et al., 1988), models that contained features of a female quail's head and neck sup- ported more copulatory responding during the training trials than did the fully artificial stimulus object (Figure 4). Copulatory responding was maintained to a significant extent as the quail features of the models were gradually covered with terrycloth (Group FP1). As a consequence, the subjects in Group FP1 were 3 times more likely to show some copulatory responding with the fully artificial model during the postconditioning test than were the subjects in Group NP1. Something about the fading procedure seemed to promote conditioning of copulatory behavior to the fully artificial model.

The greater efficacy of the fading procedure may have been due solely to the use during training of models that contained female quail features. Alternatively, gradually covering those quail features as training proceeded may have been important for the development of the behavior. We also do not know to what extent the development of copulatory responding in these procedures was a function of exposing subjects to the models just before access to a live sexually receptive female. Experiments 2 and 3 were conducted to explore these issues.

\section{EXPERIMENT 2}

Experiment 2 was conducted to replicate the findings obtained in Experiment 1 with a fading sequence of models and to determine whether the copulatory behavior generated by that procedure is a result of a direct association between the models and copulatory opportunity. Two groups of male quail (paired and unpaired) were tested. Both received training with a fading sequence of models. For the paired group, exposure to a model on each trial was followed immediately with access to a live sexually receptive quail hen. For the unpaired group, exposure to a model and access to a quail hen were provided on alternate days so that presentation of the model would not come to signal impending copulatory opportunity.

\section{Method}

Subjects. Twenty male Japanese quail, 4-5 months old at the start of the experiment, served as subjects, together with an equal number of adult female birds. The birds were reared and maintained as in Experiment 1

Apparatus. The experimental chambers used in Experiment 1 were modified with the addition of a $14 \times 16.5 \mathrm{~cm}$ side door through which a female quail could be placed into the experimental chamber when access to a female was provided unpaired with exposure to a stimulus object. The door was to the right of the holding compartment in which the male subject was placed at the start of each trial of exposure to a stimulus object, $10 \mathrm{~cm}$ from the back wall and $15 \mathrm{~cm}$ above the floor (see $\mathrm{d}$ in Figure 2).

Procedure. As in Experiment 1, the subjects were assigned to one of two squads of 10 subjects each, and each squad was housed in the experimental chambers during alternate $24-\mathrm{h}$ periods. The alternating housing procedure was started 13 days before the first conditioning trial. During this preliminary phase, the subjects received access to a female quail for $5 \mathrm{~min}$ on each of four occasions and were also habituated to being placed in the side compartments and released into the test arenas, and to having the female release boxes operated.

After the preliminary procedures, the subjects were assigned to one of two groups, each of which received a fading sequence of 
stimulus objects. The subjects in Group FP2 (Fading, Paired, Experiment $2, n=10$ ) were exposed to a stimulus object for $30 \mathrm{sec}$ on each conditioning trial, immediately before receiving access to a quail hen for $5 \mathrm{~min}$. Conditioning trials were conducted every other day. The subjects in Group FU2 (Fading Unpaired Experiment $2, n=10$ ) received exposure to the stimulus objects on the same days as did the subjects in Group FP2. However, for Group FU2, access to a quail hen was provided for $5 \mathrm{~min} 1$ day after each exposure to a stimulus object. The female bird was placed into the test chambers through the side door (see $d$ in Figure 2) for the unpaired female presentations. Groups FP2 and FU2 received a fading sequence of stimulus objects similar to what was used with Group FP1, with two small exceptions. During the first two conditioning trials, the stimulus object consisted of the entire body of a taxidermically prepared quail hen positioned in a squatting posture. In addition, a stimulus object with $75 \%$ of the neck covered with terrycloth was added between Objects 2 and 3 (see Figure 1). The decision rule for advancing from one model to the next was the same as for Group FP1 in Experiment 1 and was instituted after the first two conditioning trials. Four subjects in Group FP2 and 3 in Group FU2 reached the end of the fading sequence of models during the conditioning phase. A test trial with Object 6, as in Experiment 1 , was conducted after 15 conditioning trials.

Videotaped records of the training and test trials were scored as in Experiment 1. The interobserver reliability correlations for the test session were $.97, .99$, and .99 , for the grab, mount, and cloacal contact responses, respectively.

\section{Results}

The frequencies of grab, mount, and cloacal contact responses observed with each group are summarized in Figure 5. (The connected points represent data obtained during the conditioning trials, and the unconnected points at the right represent data obtained during the postconditioning test.) Responding to the stimulus models started at a much higher level in Experiment 2 than in Experiment 1. This was probably due to the fact that during the first two trials in Experiment 2, the stimulus object consisted of the entire body of a taxidermically prepared quail hen. Previous research had shown that the entire body of a taxidermically prepared quail hen elicits a higher frequency of copulatory responses than does a model that only contains a hen's head and neck (Domjan, 1990). A head-andneck model had been used during the initial trials of Experiment 1.

Copulatory responding was maintained in the fading paired group (FP2) as conditioning progressed. The subjects in the fading unpaired group (FU2) responded as frequently as did the subjects in the paired group through the 10th trial. However, as the quail features of the stimulus objects were further covered up after Trial 10 , responding in Group FU2 declined a bit.

Separate two-way (groups $\times$ trials) ANOVAs were calculated for each response measure for the 15 conditioning trials. The analyses failed to reveal any significant main effects of trials or of groups $\left(F_{s}<1.2\right)$. However, the interaction between groups and trials was significant for each response measure $[F \mathrm{~s}(14,252)=3.10,2.67$, and 1.99 , for the grab, mount, and cloacal contact responses, respectively, all $p s<.05]$. These results indicate that the divergence of responding that occurred between Groups FP2 and FU2 toward the end of the conditioning phase was significant.
Grabs
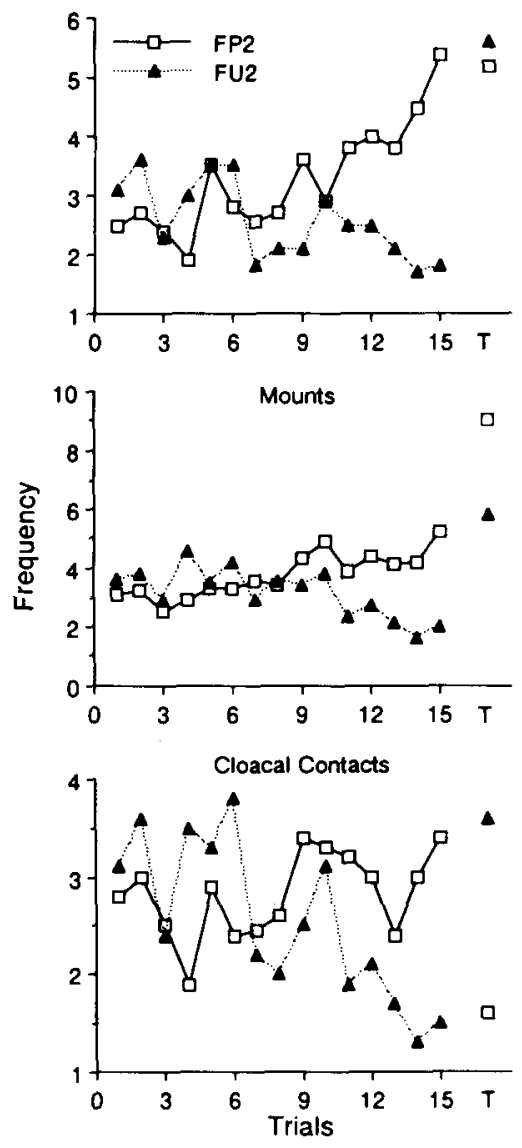

Figure 5. Mean frequencies of grab, mount, and cloacal contact responses elicited by the stimulus objects during the 15 conditioning trials (connected points) and the postconditioning test (the unconnected points on the right of each panel) in Experiment 2. (Group FP2 received a fading paired procedure; Group FU2 received a fading unpaired procedure.)

The group differences that developed toward the end of the conditioning phase were not evident in the postconditioning test session, when all of the subjects were tested with the stimulus object that was entirely covered with terrycloth (see unconnected points in Figure 5). Although there were some variations in the mean frequencies of grab, mount, and cloacal contact responses when the test session was introduced, these variations were not statistically significant. The results with the cloacal contact response were a bit peculiar because the mean response of Group FU2 increased from Conditioning Trial 15 to the test session, whereas the response of Group FP2 decreased. The high frequency of cloacal contacts in Group FU2 during the test session was primarily the result of 1 unusual subject that responded 27 times.

As in Experiment 1, the transfer of responding to the test session was evaluated for each response measure with separate ANOVAs that compared Trial 15 of conditioning and the test session. These analyses failed to reveal any significant effects of groups, the shift from conditioning to the test session, or interactions between these two 
factors (all $F \mathrm{~s}<2.5$, all $p \mathrm{~s}>$.10). Separate comparisons between Groups FP2 and FU2 of responding during the postconditioning test session for each response measure with $t$ tests also failed to reveal any significant differences (all $t s<1.0$ ).

The proportion of subjects in each group that made at least one grab, mount, and cloacal contact response during the postconditioning test session is summarized in Table 1 . Similar numbers of subjects in each group made at least one grab, mount, and cloacal contact response during the test trial with the entirely artificial object. Of the total of 20 subjects, 11 made at least one grab response to the artificial object, 10 made at least one mount response, and 8 made at least one cloacal contact response.

\section{Discussion}

The present findings confirm the observation in Experiment 1 that nearly half of the male quail exposed to a fading sequence of stimulus objects end up making copulatory responses to a stimulus object that lacks the speciestypical features of a quail hen. The present findings also help to characterize some of the boundary conditions for this phenomenon.

There was some evidence toward the end of the conditioning phase that the subjects given exposure to stimulus objects paired with access to a live quail hen (Group FP2) responded more vigorously to the objects than did the subjects that received access to a quail hen in an unpaired fashion (Group FU2). However, substantial numbers of grab, mount, and cloacal contact responses were also observed in the unpaired group. Furthermore, when all of the subjects were given a standard 5-min test with the entirely covered stimulus object at the end of the experiment, there was no evidence that a history of paired exposure to the objects significantly increased the likelihood that the subjects would copulate with the fully covered object. This finding indicates that a fading sequence of stimulus objects can result in copulatory behavior even if the objects are not signals for impending access to a live quail hen.

The absence of a clear effect of pairings suggests that copulation with the fully covered model did not develop because of a direct association of the stimulus objects with copulatory reinforcement. Several factors may have been responsible for this outcome. One possibility is that a direct association between the stimulus objects and sexual reinforcement did not develop. Exposure to the stimulus objects was not uniquely predictive of sexual reinforcement. The release of the female quail at the end of each conditioning trial was preceded by the subject being released from the holding compartment and encountering all of the stimuli of the test cage. Rather than the stimulus objects, various stimuli, including spatial cues characteristic of the area near where the female was released, could have become associated with access to the female. Another possibility is that direct associations between the stimulus objects and sexual reinforcement developed during the course of conditioning for the paired group. How- ever, such associations may not have been manifested in copulatory behavior.

\section{EXPERIMENT 3}

The results of Experiments 1 and 2 indicated that exposure to a fading sequence of stimulus objects results in nearly half of the subjects displaying copulatory behavior in response to a fully artificial stimulus object. Experiment 1 provided suggestive evidence that an important aspect of the fading procedure involved exposing subjects to models that contained the shape and plumage of a quail hen's head and neck. The subjects that received only the fully artificial model during training (Group NP1) were one third as likely to copulate with the fully artificial model as were the subjects that received the fading sequence of stimulus objects (Group FP1). However, we do not know whether gradually covering the quail features during the course of training was necessary to get the subjects to copulate with a fully artificial model. Perhaps practice in copulating with an inanimate object that has quail features was sufficient.

Experiment 3 was conducted to see if exposure to models containing natural quail features is sufficient for the development of responding to a fully artificial stimulus object. In Experiment 3, the subjects were exposed to models containing quail features, but those features were not covered up gradually during the course of training.

\section{Method}

Subjects. Sixteen male Japanese quail, 4-5 months old at the start of the experiment, served as subjects, along with an equal number of sexually receptive adult female quail. The subjects were reared and maintained as in Experiments 1 and 2. Three of the subjects failed to copulate with live quail hen in the experimental chambers and were therefore omitted from the study.

Apparatus. The experimental chambers that were used in Ex periment 1 were also used in Experiment 3.

Procedure. As in Experiment 1, the subjects were assigned to one of two squads (of 8 subjects each), and each squad was housed in the experimental chambers during alternate 24-h periods. The alternating housing procedure was started 27 days before the first conditioning trial. During this preliminary habituation phase, the subjects received access to a female quail for $5 \mathrm{~min}$ on each of eight occasions to facilitate their responding to inanimate objects (Domjan et al., 1989). They were also habituated to being placed in the side compartments and released into the test arenas, and to having the female release boxes operated.

The conditioning procedure was similar to that used with the nonfading group in Experiment 1 . Trials consisted of permitting the subject to enter the test arena with a stimulus object available under the female release box. Thirty seconds later, a live quail hen was released into the test arena for $5 \mathrm{~min}$. On Trials 1-6 and 9-18, Stimulus Object 1 (Figure 1) was used, which had a taxidermically prepared female quail head and neck. On Trials 7 and 8, the stimulus object consisted of the entire body of a quail hen taxidermically prepared and positioned in a squatting posture. After Trial 18, the standard 5 -min test trial with Object 6 , which was entirely covered with terrycloth, was conducted, as in Experiments 1 and 2. Interobserver reliability correlations for the test session were $.94, .91$, and 1.0 , for the grab, mount, and cloacal contact responses, respectively. 


\section{Results}

The frequencies of grab, mount, and cloacal contact responses directed toward the stimulus objects during the training trials are summarized by the connected points in Figure 6. The subjects gradually increased responding during Trials 1-6 when they received exposure to a stimulus object containing just the head and neck of a quail hen. Responding increased substantially during Trials 7 and 8 , when the stimulus object consisted of the entire body of a quail hen, but returned to lower levels when the head-and-neck model was returned for Trials 9-18.

Although substantial levels of copulatory responding $\alpha$ curred during the training phase, the copulatory behavior was not maintained when the fully artificial model was introduced during the postconditioning test trial. Only 1 of the 13 subjects made a cloacal contact response with the fully artificial model (see Table 1). The frequencies of grab, mount, and cloacal contact responses that occurred in response to the fully artificial model are represented by the unconnected points in Figure 6. A comparison of responding during the postconditioning test and the last trial of the training phase indicated that significantly fewer copulatory responses occurred in response
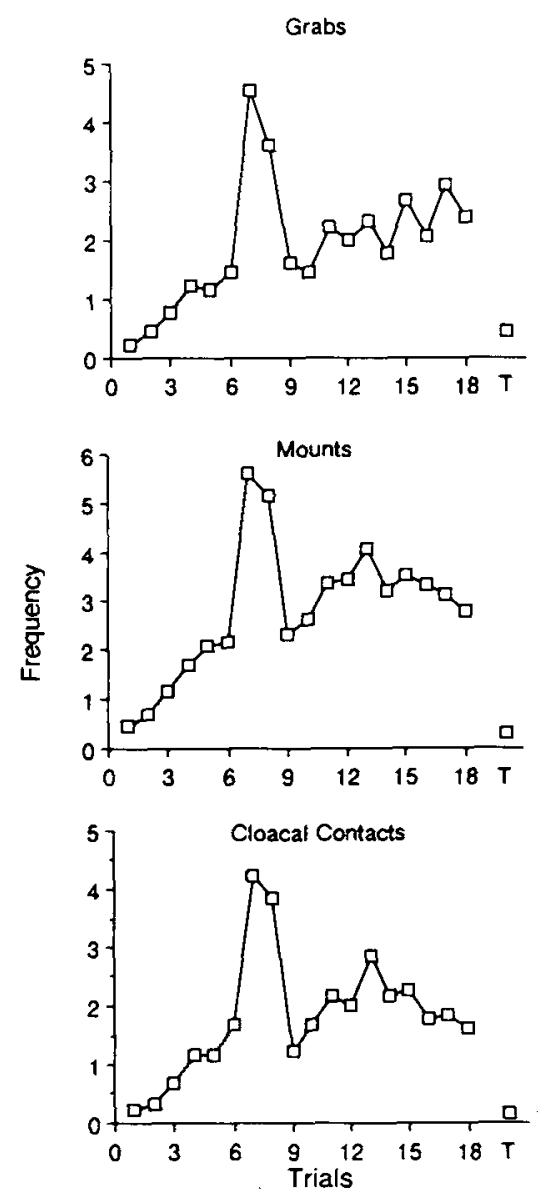

Figure 6. The mean frequencies of grab, mount, and cloacal contact responses elicited by the stimulus objects during the 18 conditioning trials (connected points) and the postconditioning test (the unconnected points on the right of each panel) in Experiment 3. to the fully artificial model than were observed at the end of the training phase, when subjects were exposed to a model containing the head and neck of a female quail $[t s(12)=3.58,2.92$, and 3.08 , for grab, mount, and cloacal contact responses, respectively, all $p$ s $<.05]$.

\section{Discussion}

Experiment 3 was conducted to explore the possibility that exposure to the fading sequence of stimulus objects in Experiments 1 and 2 resulted in high levels of copulation with the fully artificial model because, during training, the fading sequence permitted copulation with models containing quail features. The subjects in Experiment 3 were exposed to a model containing the head and neck of a female quail on Trials $1-6$ and 9-18 and received exposure to a full-body quail model on Trials 7 and 8 . Despite considerable experience with and copulatory responding to models containing quail features during training, the subjects in Experiment 3 failed to maintain their responding when the fully artificial model was introduced during the postconditioning test session. This result contrasts with the behavior of the subjects in the fading groups in Experiments 1 and 2, which maintained their copulatory responding without significant decline during the standard postconditioning test. Thus, the present results provide no support for the hypothesis that mere exposure to models containing quail plumage is sufficient to generate high levels of responding to a fully artificial stimulus object. Evidently the fading procedures were more successful in stimulating copulation with a fully artificial object because they involved gradually covering up the quail features during the course of training.

The present experiment did not include a replication of the fading procedures that were used in Experiments 1 and 2. However, it is unlikely that the low level of copulation observed during the postconditioning test was due to a poor set of subjects serving in the experiment. As in Experiments 1 and 2, only the subjects that reliably copulated with the live female quail during the training trials were included in Experiment 3. In addition, aspects of the training procedure in Experiment 3 that were similar to the procedures tested in Experiments 1 and 2 yielded similar performance. For example, the fading group of Experiment 1 and the subjects in Experiment 3 were both exposed to the head-and-neck model during the first five training trials. In both experiments, the subjects made few grab, mount, and cloacal contact responses on Trial 1 but increased their responding to the head-andneck model during Trials 2-5 (cf. Figures 4 and 6). A direct comparison of the data for Trials 1-5 for the fading group of Experiment 1 and the subjects in Experiment 3 yielded a significant effect of trials $[F \mathrm{~s}(4,112)=$ $4.99,9.42$, and 8.74 , for the grab, mount, and cloacal contact responses, respectively, all $p s<.01]$. However, the effects of Experiment 1 versus Experiment 3 and the trials $\times$ experiment interaction were not significant (highest $F=2.42$, all $p \mathrm{~s}>.05$ ).

On Training Trials 1 and 2 of Experiment 2, the subjects were exposed to a taxidermic model consisting of 
the entire body of a quail hen. A whole-body model was also used on Training Trials 7 and 8 in Experiment 3. To check that the subjects in Experiment 3 were just as responsive to the whole-body model as were the subjects in Experiment 2, copulatory behavior on Trials 7 and 8 of Experiment 3 was compared to behavior on Trials 1 and 2 of Experiment 2. A separate two-factor ANOVA (experiment $\times$ trials) was calculated for each response measure. No significant effects of trials, experiment, or trials $\times$ experiment interactions were obtained for the grab or cloacal contact responses (largest $F=2.76$, all ps $>$.10). Analyses of the data for the mount response indicated that the subjects in Experiment 3 responded more vigorously to the whole-body model than did the subjects in Experiment $2[F(1,31)=4.92, p<.05]$. Thus, there was no indication that the subjects in Experiment 3 were less likely to copulate with the whole-body model than were the subjects in Experiment 2 (cf. Trials 7 and 8 of Figure 6 with Trials 1 and 2 of Figure 5). These findings provide further evidence that the subjects of Experiment 3 were just as sexually responsive as were the subjects in the earlier experiments. Therefore, the failure of these subjects to show substantial copulatory responding to the fully artificial model cannot be attributed to a general lack of sexual responsiveness.

\section{GENERAL DISCUSSION}

Appetitive components of sexual behavior have been successfully conditioned to stimuli ranging from localized lights to a small stuffed toy (Domjan et al., 1986; Domjan et al., 1988; Hollis et al., 1989). In contrast, the acquisition of consummatory sexual behavior was not observed when an arbitrary stimulus object served as the conditioned stimulus in comparable sexual conditioning procedures (e.g., Domjan et al., 1988). Some investigators had reported isolated observations of avian and mammalian species copulating with artificial stimuli (Barraud, 1953; Beach, 1950; Ficken \& Dilger, 1960; Michael, 1961; Young, 1949). However, these reports involved small numbers of subjects and did not identify the training history that might have been necessary for the development of copulation with artificial objects.

The present experiments demonstrated copulation with an arbitrary stimulus object in a sizable number of male Japanese quail and helped to identify a set of experiential factors that seem to promote such behavior. We attempted to condition copulatory behavior to an arbitrary stimulus object by taking advantage of the fact that sexually experienced male Japanese quail will copulate with inanimate objects that include the head and neck of a female quail (e.g., Domjan et al., 1989). Our strategy was to establish copulation with the head-and-neck object and then gradually cover up the quail features with an arbitrary terrycloth material as training progressed.

The fading procedure was more successful than the nonfading procedures in establishing copulatory behavior to a stimulus object made entirely of terrycloth. Of the 37 subjects that were exposed to the fading procedure in Ex- periments 1 and 2,16 subjects (43\%) ended up making at least one cloacal contact response when tested with the entirely terrycloth object. In contrast, only 4 of $30(13 \%)$ subjects exposed to a nonfading procedure (Groups NPI and NP3) ended up making at least one cloacal contact response to the fully covered model. The difference between the fading and nonfading procedures in the proportion of subjects copulating with the fully covered model was significant $\left(\chi^{2}=5.72, p<.05\right)$.

Several different versions of the fading procedure were used during the course of the experiments (Groups FP1, FP2, FU2). Direct comparisons of Groups FP1, FP2, and FU2 indicated that these groups showed similar levels of responding to the fully covered model during the postconditioning test in every response measure $[F \mathrm{~s}(2,34)<$ 1.0]. We also tested two different nonfading procedures. In one (NP1), the subjects were exposed to the fully artificial model on every trial; in the other (NP3), the subjects were exposed to models containing the head and neck of a female quail on every training trial, without having these quail features gradually removed. The two nonfading groups (NP1 and NP3) showed similar levels of responding to the fully covered model during the postconditioning test in every response measure $[F \mathrm{~s}(1,28)<1.90]$.

Because the fading and nonfading groups did not differ among each other during the postconditioning test, the test data for each set of groups were pooled. ${ }^{1}$ These pooled data are summarized in Figure 7 . No significant differences were observed between the fading and nonfading groups in the frequency of pecking or in the time spent near the stimulus object during the test session $[t \mathrm{~s}(65)=$ .09 and .32 , respectively, both $p s>.70$ ]. However, the subjects that received a fading procedure made significantly more grab, mount, and cloacal contact responses to the fully covered model than did the subjects that received one of the nonfading procedures $[t \mathrm{~s}(65)=2.34$, 2.30 , and 2.19 , all $p s<.05]$. Thus, the advantages provided by a fading procedure were limited to the consummatory components of the subjects' copulatory behavior.

The present findings help to rule out several possible interpretations of the higher level of copulatory responding that occurred in the subjects that received a fading procedure. For example, the superior performance of the fading groups cannot be attributed to sensitization effects of copulatory experience with live female quail because the nonfading groups received comparable sexual experience with live quail hen.

A related interpretation may be developed on the basis of the presence of sexually conditioned contextual or other stimuli. Studies with avian, rodent, and fish species have shown that sexually conditioned stimuli increase the effectiveness of female cues in eliciting both approach and copulatory components of sexual behavior (see Domjan, 1992, for a review). One possible interpretation of these findings is that sexually conditioned stimuli decrease the threshold for eliciting copulatory behavior. Domjan et al. (1989), for example, demonstrated that male quail are more likely to copulate with an inanimate model containing only the head and neck of a female if the model is 


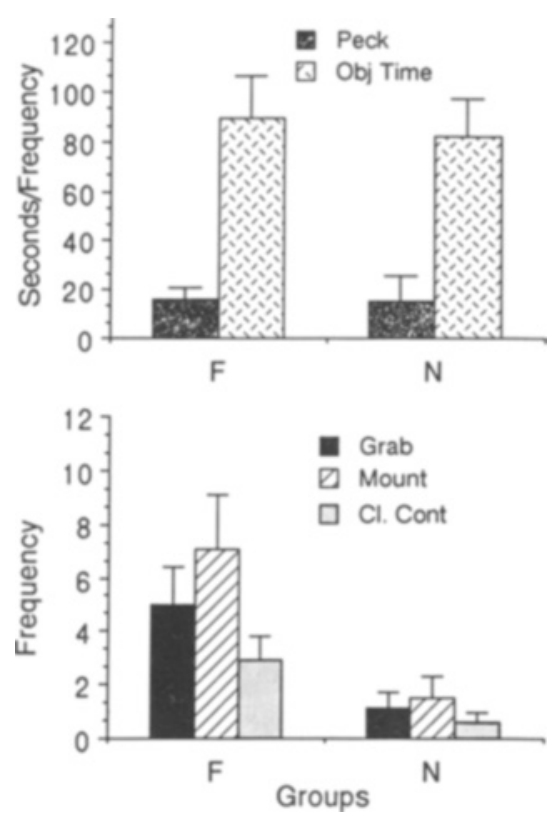

Figure 7. Top panel: Mean seconds spent near the stimulus object, and frequency of pecks at the object, during the postconditioning test trial for the fading and nonfading groups combined (right panel). Bottom panel: Mean frequencies of grab, mount, and cloacal contact responses during the postconditioning test trial for the fading and nonfading groups combined (right panel). (Vertical bars represent standard error of the mean.)

presented in a sexually conditioned context than if the model is presented in a familiar, but nonsexual, context. In the extreme, the threshold for eliciting a form of species-typical behavior may be lowered to such an extent that the behavior occurs in the absence of its usual eliciting stimulus-what ethologists have called "vacuum activity" (e.g., Ewert, 1980).

In the present studies, the contextual cues of the experimental chambers probably became associated with sexual reinforcement because the subjects received repeated opportunities to copulate with quail hen in those chambers (cf. Domjan et al., 1989; Domjan, Akins, \& Vandergriff, 1992). These conditioned contextual cues may have lowered the threshold for eliciting copulatory behavior. However, the effectiveness of the fading procedures in promoting copulation with the entirely artificial stimulus object cannot be attributed to such threshold lowering because the nonfading procedures involved similar copulatory experiences in the test chambers and therefore similar opportunities for contextual conditioning.

The threshold for eliciting sexual behavior also may have been lowered by exposure to discrete stimuli that signaled impending copulatory opportunity. For example, the subjects in the paired groups were placed in the side cage for about $5 \mathrm{~min}$ before being released back into the experimental chamber and receiving access to a live quail hen. Therefore, some aspect of being placed in the side cage and then being let back into the experimental chamber may have become a conditioned stimulus for copulatory opportunity. However, the presence of such condi- tioned stimuli cannot explain the facilitating effects of the fading procedures because the two nonfading groups (NP1 and NP3) also received copulatory opportunity paired with release from the side cages during the conditioning trials.

Another possible interpretation is that copulation with the artificial object was a form of "displacement behavior" or "interruptive activity" (e.g., Baerends, 1988; Ficken \& Dilger, 1960) that is evident when the execution of an aroused activity is thwarted. For the subjects in Groups FP1 and FP2, for example, copulatory opportunity with a live quail hen was always provided after release from the side box and exposure to a stimulus object, but presentation of the live hen was delayed for $30 \mathrm{sec}$ after the start of a trial. The thwarting of sexual behavior during this $30-\mathrm{sec}$ waiting period may have caused "displacement" copulations directed toward the stimulus objects. However, such "displacement" copulations also should have occurred in the nonfading groups NP1 and NP3, which also had to wait $30 \mathrm{sec}$ after the start of each conditioning trial to gain access to a live hen. Therefore, interpretations based on the concept of displacement behavior also do not provide a successful explanation of the differences that were obtained between the fading and nonfading groups. In addition, a displacement mechanism should not have been operative for the subjects that received the fading unpaired procedure in Experiment 2 (Group FU2), because in that procedure, the live hen was always introduced into the test chamber unannounced during the conditioning phase, on days that alternated with exposure to the stimulus objects. Nevertheless, Group FU2 did not make significantly fewer grab, mount, or cloacal contact responses during the postconditioning test than did Group FP2.

The subjects that received the fading procedures were more likely to copulate with the stimulus objects during the conditioning trials than were the subjects in Group NP1, which received the entirely artificial object on every trial (see Figure 4). The fading groups also spent more time near the stimulus objects during the conditioning trials than did Group NP1 (see Figure 3). However, these differences probably were not responsible for the differences between fading and nonfading groups that were observed during the test session. Group NP3, which received access to a stimulus object containing the head and neck of a quail hen on each conditioning trial, also copulated with the object much more often during the conditioning trials than did Group NP1 (see Figure 6). Nevertheless, Group NP3 did not show much copulatory behavior during the test session. The independence of time spent near a stimulus object and copulation is evident in the fact that the fading and nonfading groups spent comparable amounts of time near the stimulus object during the test session but showed significantly different degrees of copulatory behavior (see Figure 7).

The subjects that received the fading procedures gained more familiarity with the terrycloth covering during the course of the conditioning trials than did Group NP3, which never had the quail head and neck covered with terrycloth (even partially) during the conditioning trials. 
However, the simple hypothesis that familiarity with the terrycloth covering promotes copulation with a terrycloth object cannot explain the obtained difference between the fading and nonfading procedures because one of the nonfading groups (NP1) received as much or more exposure to the terrycloth covering as did the fading groups.

Other interpretations of the difference between the fading and nonfading groups are based on presumed special properties of the quail features that were present in the stimulus objects used in the fading procedures. For example, because of the special significance of quail features in eliciting sexual behavior, the presence of quail features may have enhanced attention to the stimulus objects and thereby promoted learning about the objects. Thus, the quail features may have enhanced processing of the terrycloth stimulus. However, for such an interpretation to be successful, it has to be elaborated to explain why the presumed increase in attention enhanced copulatory responding but did not enhance pecking and time spent near the object during the postconditioning test.

Because Group FU2 received access to a live hen on days alternating with exposure to the stimulus objects, the results obtained with Group FU2 make unlikely any explanation that assumes that the fading procedures somehow enhanced a direct association between the stimulus objects and reinforcement obtained from copulation with a live hen. The high frequency of copulatory responses observed during the test session with Group FU2 suggests an associative mediational interpretation of the effectiveness of the fading procedure (Holland, 1981). This interpretation assumes the learning of two associations. One is presumably formed during exposure to a stimulus object and involves an association between the quail features of the stimulus objects and the terrycloth covering. The second association is presumably formed during copulatory experience with a live quail and involves the association of visual features of the hen's head and neck with copulatory reinforcement. For these two associations to be formed, exposure to the stimulus objects does not have to be paired with copulatory opportunity. The presence of similar quail features in the stimulus objects and the live quail hen serves to mediate an association between the terrycloth covering and sexual reinforcement.

All of the fading procedures that were used allowed for the operation of the associative mediation mechanism. In contrast, the mechanism could not operate with the nonfading procedure that involved exposure to a fully covered model on every conditioning trial (Group NP1). Therefore, the associative mediation mechanism is consistent with the poor performance of Group NP1 during the test session. The mechanism provides a different account of the poor performance of Group NP3, which was exposed to an object that included a quail head and neck on every conditioning trial. The stimulus objects that were used with Group NP3 had the quail features that are needed to activate the mediation mechanism. However, the headand-neck stimuli were not covered up during the course of conditioning. Therefore, the terrycloth covering parts of the neck and head of the stimulus objects could not become conditioned. The poor performance of Group NP3 presumably reflected the lack of opportunity for conditioning of the terrycloth on the vertical section of the entirely artificial stimulus object (see Figure 1).

The associative mediation interpretation assumes that copulatory experience with live female quail is important for the efficacy of the fading procedure. This assumption was not tested in the present experiments. However, prior research has shown that copulatory experience with live quail hen facilitates copulation with a head-and-neck model (Domjan et al., 1989). Consistent with that outcome, very little copulatory behavior was observed in the present experiments when the subjects were exposed to a headand-neck model during the first few conditioning trials, before they had much copulatory experience with live quail hen (see Group FP1 in Figure 4, and Group NP3 in Figure 6). Given these findings, it is unlikely that significant copulatory behavior would have developed in the fading groups without copulatory experience with live quail hen.

The present experiments do not permit specification of the form of associative mediation that might have been operative in the fading groups. Since we intermixed exposures to the stimulus objects and copulation with live female quail, our procedures did not follow the protocol for either sensory preconditioning or second-order conditioning. Our fading procedures may have activated one or both of these mechanisms.

Any complete account of the present findings has to provide an explanation of the dissociation that was observed between the copulatory and noncopulatory response measures. Although the fading groups were more likely than the nonfading groups to engage in copulatory behavior during the test session, the two types of groups spent similar amounts of time near the stimulus object and showed similar levels of pecking behavior. Pecking may not have reflected sexually conditioned behavior (Schlinger, Palter, \& Callard, 1987). Consistent with that interpretation, acquisition effects were not evident with the pecking response (see Experiment 1). In contrast, time spent near a conditioned stimulus frequently has been used as a measure of sexual conditioning and has been shown to reflect associative processes (e.g., Domjan et al., 1986; Domjan et al., 1988). The nonfading groups may have spent as much time near the stimulus object as did the fading groups because for Groups NP1 and NP3, the area around the stimulus object was also the area where the live female was released into the experimental chamber during conditioning trials (see Figure 2). Therefore, the time the nonfading groups spent in this area may have reflected the conditioning of spatial cues rather than the conditioning of the stimulus object. Given these considerations, the fading and nonfading groups may have shown similar time scores during the test session for different reasons. The approach behavior of the fading groups may have been controlled primarily by the stimulus object, whereas the 
approach behavior of the nonfading groups may have been controlled by the place where the female quail had been presented during conditioning trials.

Another possibility is that the approach behavior became conditioned to features of the artificial stimulus object in both the fading and the nonfading groups but that the conditioning of copulatory behavior required more stringent conditioning parameters than did the conditioning of approach behavior. This alternative is consistent with earlier characterizations of appetitive behavior as being more flexible than consummatory behavior (Eibl-Eibesfeldt, 1970; Tinbergen, 1951). Such differences in susceptibility to learning may be related to temporal variables. Timberlake and Lucas (1989), for example, have suggested that shorter CS-US intervals are required for the conditioning of consummatory components of behavior sequences rather than for the conditioning of appetitive responses. The fading procedures involved simultaneous pairings between the quail plumage and terrycloth features of the stimulus objects. Such temporal contiguity may have been more important for the conditioning of copulatory responses than for the conditioning of approach behavior.

\section{REFERENCES}

AlleN, A. A. (1934). Sex rhythm in the ruffed grouse (Bonasa umbellus) and other birds. Auk, 51, 180-199.

Baerends, G. P. (1988). Ethology. In R. C. Atkinson, R. J. Hermstein, G. Lindzey, \& R. D. Luce (Eds.), Stevens' handbook of experimental psychology (2nd ed., Vol. 1, pp. 765-830). New York: Wiley.

Barraud, E. M. (1953). Sexual behavior occurring as overflow activity in juvenile house sparrow. British Birds, 46, 382.

BEACH, F. A. (1950). Sexual behavior in animals and man. The Harvey Lectures, 43, 259-279.

Carbaugh, B. T., Schein, M. W., \& Hale, E. B. (1962). Effects of morphological variations of chicken models on sexual responses of cocks. Animal Behaviour, 10, 235-238.

Domjan, M. (1990). Modification of sexual behavior through conditioning: An avian model. In J. R. Feierman (Ed.), Pedophilia: Biosocial dimensions (pp. 242-273). New York: Springer-Verlag.

Domsan, M. (1992). Adult learning and mate choice: Possibilities and experimental evidence. American Zoologist, 32, 48-61.

Domian, M., Akins, C., \& VandergrifF, D. H. (1992). Increased responding to female stimuli as a result of sexual experience: Tests of mechanisms of learning. Quarterly Journal of Experimental Psychology, 45B, 139-157.

Domina, M., Greene, P., \& NorTh, N. C. (1989). Contexual conditioning and the control of copulatory behavior by species specific sign stimuli in male Japanese quail. Journal of Experimental Psychology: Animal Behavior Processes, 15, 147-153.

Domian, M., Lyons, R., North, N. C., Bruell, J. (1986). Sexual Pavlovian conditioned approach behavior in male Japanese quail (Cotumix coturnix japonica). Joumal of Comparative Psychology, 100, 413-421.

Domjan, M., Nash, S. (1988). Stimulus control of social behaviour in male Japanese quail (Coturnix cotumix japonica). Animal Behaviour, 36, 1006-1015.

Domjan, M., O'VAry, D., \& Greene, P. (1988). Conditioning of ap- petitive and consummatory sexual behavior in male Japanese quail. Journal of the Experimental Analysis of Behavior, 50, 505-519.

EIBL-EIBESFELDT, I. (1970). Ethology: The biology of behavior. New York: Holt, Rinehart \& Winston.

EWERT, J.-P. (1980). Neuroethology. Berlin: Springer-Verlag.

FICKEN, M. S., \& Dulger, W. C. (1960). Comments on redirection with examples of avian copulations with substitute objects. Animal Behaviour, 8, 219-222.

Holland, P. C. (1981). Acquisition of representation-mediated conditioned food aversions. Learning \& Motivation, 12, 1-18.

Hollis, K. L., Cadieux, E. L., Colbert, M. M. (1989). The biological function of Pavlovian conditioning: A mechanism for mating success in the blue gourami (Trichogaster trichopterus). Journal of Comparative Psychology, 103, 115-121.

MiCHAEL, R. P. (1961). "Hypersexuality" in male cats without brain damage. Science, 134, 553-554.

NoBlE, G. K., \& VOGT, W. (1935). An experimental study of sex recognition in birds. Auk, 52, 278-286.

Schein, M. W., Diamond, M., \& Carter, C. S. (1972). Sexual performance levels of male Japanese quail (Coturnix coturnix japonica). Animal Behaviour, 20, 61-66.

SCHEIN, M. W., HALE, E. B. (1965). Stimuli eliciting sexual behavior. In F. Beach (Ed.), Sex and behavior (pp. 440-482). New York: Krieger.

Schlinger, B. A., Palter, B., \& Callard, G. V. (1987). A method to quantify aggressiveness in Japanese quail (Coturnix corumix japonica). Physiology \& Behavior, 40, 343-348.

Schoettle, H. E. T., Schein, M. W. (1959). Sexual reactions of male turkeys to deviations from a normal female head model. Anatomical Record, 134, 635.

Terrace, H. S. (1966). Stimulus control. In W. K. Honig (Ed.), Operant behavior: Areas of research and application (pp. 271-344). New York: Appleton-Century-Crofts.

Timberlake, W., LuCas, G. A. (1989). Behavior systems and learning: From misbehavior to general principles. In S. B. Klein \& R. R. Mowrer (Eds.), Contemporary learning theories: Instrumental conditioning theory and the impact of biological constraints on learning (pp. 237-275). Hilisdale, NJ: Erlbaum.

TiNBERGEN, N. (1951). The study of instinct. London: Oxford University Press.

Wilson, M. I., Bermant, G. (1972). An analysis of social interaction in Japanese quail (Coturnix coturnix japonica). Animal Behaviour, 20, 252-258.

YouNG, H. (1949). Atypical copulatory behavior of a robin. Auk, 66, 94.

\section{NOTE}

1. The three experiments were conducted consecutively, without a break. Although the experiments involved birds from different hatchings at slightly different times of year, the subjects were maintained under standard laboratory conditions, which should have resulted in comparable sexual motivation. To further insure comparability of the subjects, the subjects selected for participation in the experiments were all pretested for copulatory behavior with a live quail hen. All aspects of the experiments that permitted direct assessment of the comparability of the subjects across experiments confirmed the absence of systematic differences. Both the procedures and results of the fading paired procedure were replicated in Experiments 1 and 2 (Groups FP1 and FP2, respectively), and the subjects in Experiment 3 responded similarly to the subjects in Experiments 1 and 2 that were exposed to the same types of models during conditioning (see Results of Experiment 3 ).

(Manuscript received July 2, 1991; revision accepted for publication April 15, 1992.) 